\title{
Association of Base Excision Repair Gene Polymorphisms with ESRD Risk in a Chinese Population
}

\author{
Zhenming Cai, ${ }^{1,2}$ Huimei Chen, ${ }^{1,2}$ Jing Tao, ${ }^{3}$ Wenwen Guo, ${ }^{1,2}$ Xiufang Liu,, 2 \\ Bixia Zheng, ${ }^{1,2}$ Wei Sun, ${ }^{3}$ and Yaping Wang ${ }^{1,2}$ \\ ${ }^{1}$ Department of Medical Genetics, Nanjing University School of Medicine, Nanjing 210093, China \\ ${ }^{2}$ Jiangsu Key Laboratory of Molecular Medicine \& State Key Laboratory of Pharmaceutical Biotechnology, Nanjing University, \\ Nanjing 210093, China \\ ${ }^{3}$ Jiangsu Province Hospital of TCM, Nanjing University of Traditional Chinese Medicine, Nanjing 210029, China
}

Correspondence should be addressed to Huimei Chen, chenhuimei@nju.edu.cn and Yaping Wang, wangyap@nju.edu.cn

Received 6 February 2012; Revised 6 April 2012; Accepted 6 April 2012

Academic Editor: Krzysztof Ksiazek

Copyright ( $) 2012$ Zhenming Cai et al. This is an open access article distributed under the Creative Commons Attribution License, which permits unrestricted use, distribution, and reproduction in any medium, provided the original work is properly cited.

\begin{abstract}
The base excision repair (BER) pathway, containing OGG1, MTH1 and MUTYH, is a major protector from oxidative DNA damage in humans, while 8-oxoguanine (8-OHdG), an index of DNA oxidation, is increased in maintenance hemodialysis (HD) patients. Four polymorphisms of BER genes, OGG1 c.977C > G (rs1052133), MTH1 c.247G > A (rs4866), MUTYH c.972G > C (rs3219489), and AluYb8MUTYH (rs10527342), were examined in 337 HD patients and 404 healthy controls. And the 8-OHdG levels in leukocyte DNA were examined in 116 HD patients. The distribution of MUTYH c.972 GG or AluYb8MUTYH differed between the two groups and was associated with a moderately increased risk for end-stage renal disease $(\mathrm{ESRD})(P=0.013$ and 0.034 , resp.). The average $8-\mathrm{OHdG} / 10^{6} \mathrm{dG}$ value was significantly higher in patients with the OGG1 c.977G, MUTYH c.972G or AluYb8MUTYH alleles ( $P<0.001$ via ANOVA). Further analysis showed that combination of MUTYH c.972GG with OGG1 c.977GG or AluYb8MUTYH increased both the risk for ESRD and leukocyte DNA 8-OHdG levels in HD patients. Our study showed that MUTYH c.972GG, AluYb8MUTYH, and combination of OGG1 c.977GG increased the risk for ESRD development in China and suggested that DNA oxidative damage might be involved in such process.
\end{abstract}

\section{Introduction}

Oxidative stress is characterized by an excess of reactive oxygen species (ROS) and leads to cellular injury via reactions with proteins, nucleic acids, and lipids [1,2]. The DNA bases, especially guanine $(G)$, are particularly susceptible to oxidation, for which ROS frequently lead to a plethora of oxidized guanine products [3]. 8-hydroxy-2' deoxyguanosine (also known as 8-oxoguanine; 8 -OHdG) is one of the most common mutagenic products and pairs with adenine in double-stranded DNA during DNA replication $[3,4]$. If the mispairing is not repaired, it will lead to a $G: C$ to $T: A$ transversion mutation in cells [5].

Several repair pathways are involved with the DNA insults that result from either endogenous sources or exogenous sources, including the direct reversal pathway, the mismatch repair (MMR) pathway, the nucleotide excision repair (NER) pathway, and the base excision repair (BER) pathway [6]. Base excision repair (BER) is the primary DNA repair pathway that corrects base lesions that arise due to oxidative, alkylation, deamination, and depurinatiation/depyrimidination damage, such as 8-OHdG [7]. Actually, the BER pathway specifically prevents those G:C-to$\mathrm{T}$ : A mutations by the repair of $8-\mathrm{OHdG}$. It includes the MTH1, OGG1, and MUTYH genes that prevent, recognize and remove the misincorporated oxidized nucleotide, 8$\mathrm{OHdG}$, and the adenine paired with $8-\mathrm{OHdG}$, respectively, when initiated by the BER pathway.

Increasing evidence has shown that genetic polymorphisms in DNA repair genes may modulate DNA repair capacity, result in DNA damage accumulation, and then contribute to some complex diseases [8, 9]. Kasahara et al. have reported that MUTYH Gln324His (c.972G > C) is associated with increased risk of colorectal cancers [10]. Marchand et al. have described the effect of OGG1 Ser326Cys (c.977C > G) on the risk of lung cancer [11]. We have also shown that 
TABle 1: Sequences of PCR Primers used for genotyping.

\begin{tabular}{|c|c|c|c|}
\hline Polymorphisms & Primer sequence $\left(5^{\prime}-3^{\prime}\right)$ & Annealing temperature $\left({ }^{\circ} \mathrm{C}\right)$ & Product length (bp) \\
\hline rs1052133: & F: $5^{\prime}$-actgtcactagtctcaccag- $3^{\prime}$ & \multirow{2}{*}{ 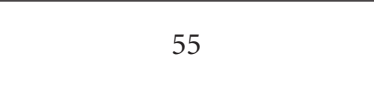 } & \multirow{2}{*}{200} \\
\hline OGG1 c.977C > G (Ser326Cys) & R: $5^{\prime}$-ggaaggtgcttggggaat- $3^{\prime}$ & & \\
\hline rs4866: & F: $5^{\prime}$-gagcggtctgacagtgga- $3^{\prime}$ & \multirow{2}{*}{58} & \multirow{2}{*}{168} \\
\hline MTH1 c.247G > A (Val83Met) & R: $5^{\prime}$-tggcactcagagatggtttg- $3^{\prime}$ & & \\
\hline rs3219489: & F: $5^{\prime}$-cccattccagttcttcctct-3' & \multirow{2}{*}{58} & \multirow{2}{*}{208} \\
\hline $\begin{array}{l}\text { MUTYH c. } 972 \mathrm{G}>\mathrm{C} \\
\text { (Gln324His) }\end{array}$ & R: $5^{\prime}$-cctttctggggaagttgacc- $3^{\prime}$ & & \\
\hline rs10527342: & F: $5^{\prime}$-tcttgacctggagaccttcc- $3^{\prime}$ & \multirow{2}{*}{60} & \multirow{2}{*}{500 or 826} \\
\hline AluYb8MUTYH & R: $5^{\prime}$-agctgcttcctccaaacagc- $3^{\prime}$ & & \\
\hline
\end{tabular}

the AluYb8 insertion in MUTYH (AluYb8MUTYH) might be a risk factor for age-related diseases and type 2 diabetes mellitus $[12,13]$.

The kidney is highly vulnerable to any of the results caused by ROS, and leukocyte 8 -OHdG content is a surrogate biomarker for oxidation-induced DNA damage in patients with end-stage renal disease (ESRD), especially those on maintenance hemodialysis (HD). Oxidative injury is thought to alter the structure and function of glomeruli and is suggested to be related to renal diseases risk and eventual ESRD as well as atherosclerosis, dialysis-related amyloidosis and anemia in incident dialysis patients $[14,15]$. The primary role of DNA repair in ESRD may be complex. Fukushima et al. [16] demonstrated that the polymorphism of the hOGG1 (Ser326Cys) was associated with progression of IgA nephropathy. Most recently, Trabulus et al. [17] showed that XRCC1 Arg399Gln polymorphism may confer increased risk for the development of ESRD in Turkey, which is the first report showing an association between DNA repair gene polymorphisms and ESRD development. However, the genetic variations involved in antioxidant defense still need to be clarified in this disease, especially in China.

Based on the association of BER polymorphisms, oxidative DNA damages, and ESRD, we hypothesized that genetic variation in the BER genes might lead to repair impairment or disability, oxidative DNA damage accumulation, and pathogenesis of ESRD. Given the potential roles of OGG1 c. $977 \mathrm{C}>\mathrm{G}$, MTH1 c. $247 \mathrm{G}>\mathrm{A}$, MUTYH c. $972 \mathrm{G}>\mathrm{C}$, and AluYb8MUTYH in the oxidative DNA repair pathway; we examined the association between these four polymorphisms in the BER pathway and ESRD in a Chinese cohort. We also assessed the leukocyte DNA 8-OHdG levels in HD patients to reveal the correlation between oxidative damage and endstage renal disease arises.

\section{Materials and Methods}

2.1. Subjects. The allelic frequency of OGG1 (NG_012106.1) c.977C > G, MTH1 (NC_000007.13) c.247G > A, MUTYH (NG_008189.1) c.972G > C, and AluYb8MUTYH (AluYb8 insertion at intron 15 of MUTYH [12]) was investigated in 337 HD patients, regardless of cause, in Nanjing, Jiangsu province, China, between October 2009 and February 2010. All patients had been maintained on hemodialysis protocols for $>3$ months and were reviewed for age, sex, and presentation of clinical and laboratory data. Hypertension was defined as systolic blood pressure (SBP) $\geq 140 \mathrm{mmHg}$ and/or diastolic blood pressure (DBP) $\geq 90 \mathrm{mmHg}$ and/or use of antihypertensive medication [18]; anemia was defined as an $\mathrm{Hgb}<11 \mathrm{~g} / \mathrm{dL}$ or use of recombinant human erythropoietin [19].

Healthy individuals with normal renal function were recruited from volunteers receiving health checkups in the same region. Detailed interview and various laboratory analyses were made upon every individual, including albumin excretion rate (AER) and serum creatinine. The subjects were excluded if their albumin excretion rate (AER) $\geq 30 \mathrm{mg} / 24 \mathrm{~h}$, serum creatinine $\geq 1.2 \mathrm{mg} / \mathrm{dL}$ and ultrasound of the kidney and ureter was abnormal in size and appearance. They were ruled out if they suffering from certain diseases, such as acute inflammation, and diabetes, hypertension, autoimmune diseases or cancer according to past history and the clinical or laboratory characteristics. A total of 404 sex and age matched subjects were selected for inclusion in the control cohort. The Institutional Ethics Committee of Nanjing University School of Medicine approved this study, and written informed consents were obtained from all participants.

2.2. High-Resolution Melting Analysis. In this study, OGG1 c. $977 \mathrm{C}>\mathrm{G}$, MTH1 c. $247 \mathrm{G}>\mathrm{A}$, and MUTYH c. $972 \mathrm{G}>\mathrm{C}$ were genotyped using the dsDNA dye LCGreen in combination with HRM analysis. DNA was extracted from peripheral blood samples, and PCR was performed to amplify the target sequences. The PCR primers were designed by LightScanner primer design software (Idaho Technology) (Table 1). Each PCR reaction was initially performed in a final reaction volume of $10 \mu \mathrm{L}$, using $25 \mathrm{ng}$ of genomic DNA, $0.2 \mathrm{pmol}$ of each primer, $0.8 \mu \mathrm{L} 2.5 \mathrm{mM}$ dNTPs, $1 \mu \mathrm{L} 25 \mathrm{mM} \mathrm{MgCl}_{2}, 1 \mu \mathrm{L}$ $10 \times$ Taq buffer with $\left(\mathrm{NH}_{4}\right)_{2} \mathrm{SO} 4,0.4 \mathrm{U}$ Taq DNA Polymerase (Fermentas), and $0.4 \mu \mathrm{L}$ dimethyl sulfoxide (DMSO). The reaction mixture was incubated at $95^{\circ} \mathrm{C}$ for $5 \mathrm{~min}$ and then subjected to 40 cycles of $95^{\circ} \mathrm{C}$ for $30 \mathrm{sec}, 55-58^{\circ} \mathrm{C}$ (Table 1) for $30 \mathrm{sec}$, and $72^{\circ} \mathrm{C}$ for $30 \mathrm{sec}$, followed by $72^{\circ} \mathrm{C}$ for $7 \mathrm{~min}$ using a PTC-200 thermal cycler (Bio-Rad).

The $9 \mu \mathrm{L}$ reaction was supplemented with $1 \mu \mathrm{L} 1 \times$ LCGreen PLUS (Idaho Technology), and the 96-well plate (Bio-Rad) was transferred to the Light Scanner (Idaho 


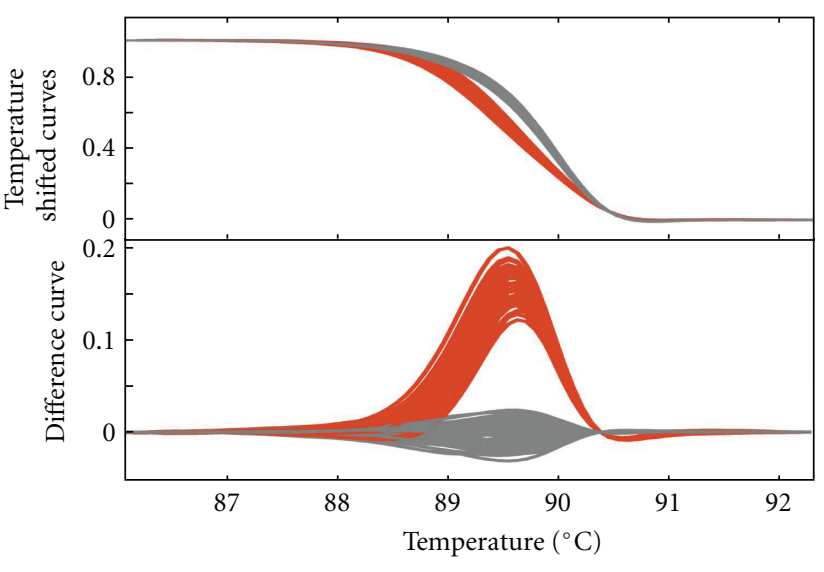

(a)

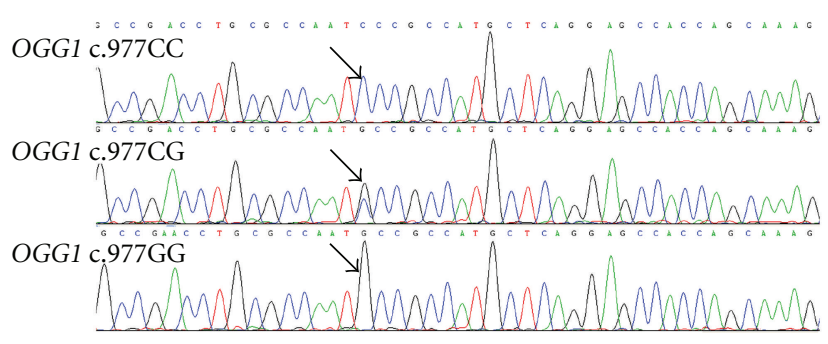

(c)

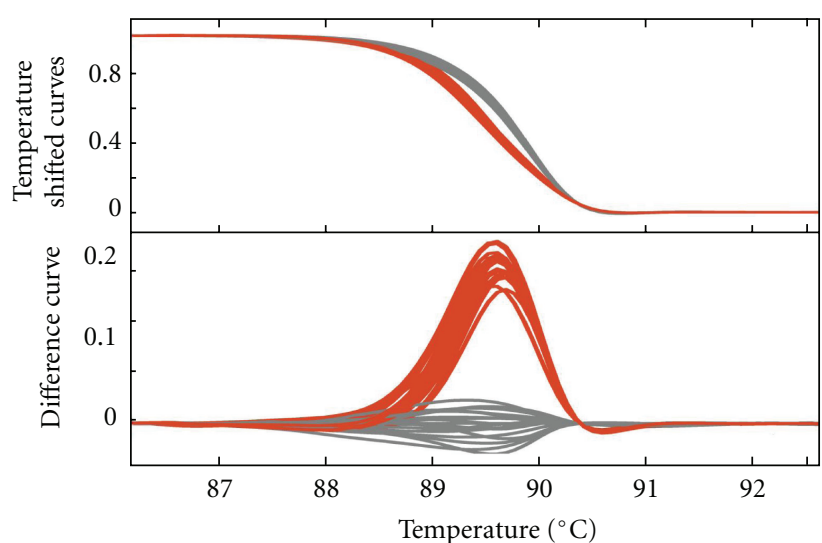

(b)

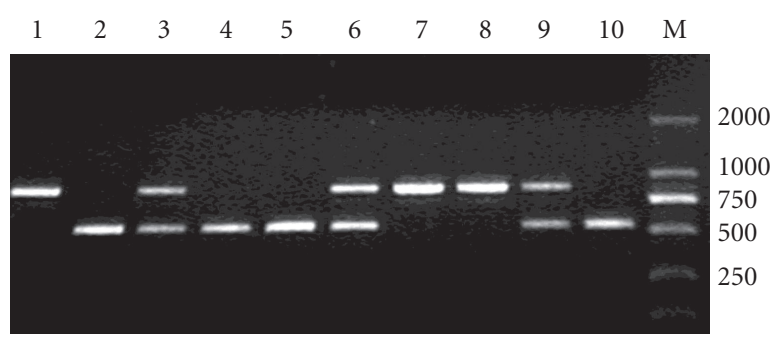

(d)

FIGURE 1: Demonstration of genotyping and sequence. (a) HRM directly discriminated the heterozygotes (OGG1 c.977 CG) and homozygotes (OGG1 c.977 CC or GG). (b) homozygous PCR products (CC or GG) were measured by LightScanner after being mixed with an equal amount of a known product (CC), which distinguished the wild homozygous samples (CC) from the variant ones (GG), as the mutational homozygotes (GG) were converted into heterozygotes (CG). (c) random samples from OGG1 c.977C > G testing were sequenced for confirmation. (d) The PCR products were separated using $1 \%$ agarose gels to assess the pattern of $A l u Y b 8$ insertion into the MUTYH gene. Lanes 2, 4, 5, and 10: absence/absence $(A / A) ; 3,6$, and 9: absence/presence $(A / P) ; 1,7$, and 8: presence/presence $(P / P)$; M: DNA Marker 2000.

Technology). Fluorescence data was collected over a temperature range of $70^{\circ} \mathrm{C}-97^{\circ} \mathrm{C}$, as the samples were melted. Melting curve analysis was performed according to the manufacturer's software. HRM could directly discriminate the heterozygote (CG) and homozygote (CC or GG) genotypes of OGG1 c. $977 \mathrm{C}>\mathrm{G}$ through melt scanning (Figure 1(a)). After mixing homozygous DNA with an equal amount of known PCR products (e.g., CC), it further distinguished between the CC and GG genotypes (Figure 1(b)). For further confirmation, $10 \%$ of samples from each group detected by HRM were randomly selected and subjected to DNA sequencing (Figure 1(c)). Similarly, the MTH1 c.247G > A and MUTYH c.972G > C polymorphisms were genotyped by HRM.

\subsection{Agarose Gel Assay for AluYb8MUTYH Polymorphism.} The PCR primers were listed in Table 1, and the PCR condition was carried out with an initial denaturation at $94^{\circ} \mathrm{C}$ for $5 \mathrm{~min}, 35$ cycles of denaturation at $94^{\circ} \mathrm{C}$ for $30 \mathrm{sec}$, annealing at $60^{\circ} \mathrm{C}$ for $30 \mathrm{sec}$, extension at $72^{\circ} \mathrm{C}$ for $50 \mathrm{sec}$, and then a final extension at $72^{\circ} \mathrm{C}$ for $10 \mathrm{~min}$. The PCR products were run out on $1 \%$ agarose gels (Invitrogen, Carlsbad, CA, USA). The AluYb8MUTYH genotypes were classified as homozygous absence of this variation (only 500 bp products, absence/absence, $A / A)$, homozygous presence of this variation (only $826 \mathrm{bp}$ products, presence/presence, $P / P$ ), and heterozygote (500 bp and $826 \mathrm{bp}$ products, absence/presence, $A / P)$, according to the variant fragment absence or presence (Figure 1(d)).

\subsection{Measurement of 8-OHdG Levels in Genomic DNA of Blood} Cells. For measuring the level of $8-\mathrm{OHdG}, 116$ patients were randomly rerecruited from the HD cohort and investigated by the method reported previously [12]. Briefly, DNA extraction from fasting venous whole blood $(10 \mathrm{~mL}$, with EDTA added to prevent coagulation) was performed within $1 \mathrm{~h}$ of collection, using the salting out method [20]. The purity of the DNA sample was checked by OD260 nm/OD280 nm and $\mathrm{OD} 260 \mathrm{~nm} / \mathrm{OD} 230 \mathrm{~nm}$ using an Eppendorf BioPhotometer Plus (Eppendorf, North America). Acceptable DNA stored frozen at $-80^{\circ} \mathrm{C}$ until all samples could be assayed at the same time.

The DNA $(200 \mu \mathrm{g})$ of each sample was dissolved in $135 \mu \mathrm{L}$ of water. Sodium acetate $(15 \mu \mathrm{L}, 200 \mathrm{mM})$ and Nuclease P1 $(15 \mu \mathrm{L}, 6$ units, Sigma, USA) were added to the DNA solution and incubated at $37^{\circ} \mathrm{C}$ for $30 \mathrm{~min}$. Tris- $\mathrm{HCl}$ buffer $(15 \mu \mathrm{L}, 1 \mathrm{M}, \mathrm{pH} 7.4)$ and alkaline phosphatase $(7 \mu \mathrm{L}, 2$ units, TAKARA, Shiga, Japan) were added and incubated at $37^{\circ} \mathrm{C}$ 
for another $30 \mathrm{~min}$. The hydrolysate was filtered through Millipore Microcon columns at $14000 \mathrm{rpm}$ for $10 \mathrm{~min}$, and $50 \mu \mathrm{L}$ of digested DNA was applied to one well of an ELISA kit (Highly Sensitive 8-OHdG Check, JaICA, Fukuroi, Shizuoka, Japan). Results were measured in nanograms per milliliter, and then $1 \mathrm{ng} / \mathrm{mL}$ was converted to 4.88 OHdG $/ 10^{6} \mathrm{dG}$ based on Halliwell [21].

2.5. Statistical Analysis. All statistical analyses were carried out using the statistical program SPSS, version 15.0. Descriptive statistical values included mean \pm SD values for continuous data and percentages for categorical data. Chisquared tests were used to compare the genotype and allelic frequencies for patients and healthy controls. Odds ratios (OR) are shown with 95\% confidence intervals (CIs). Separate comparisons of variables among subjects with different genotypes were conducted with ANOVA and followed by post hoc analysis. Since 8-OHdG levels in leukocyte DNA were positively skewed, a natural logarithmic transformation was used to normalize the distributions for analyses. In all cases, a $P$ value of less than 0.05 was considered statistically significant.

\section{Results}

Of the $337 \mathrm{HD}$ patients, $212(62.9 \%)$ were men, and 125 $(37.1 \%)$ were women. The average age was $53.1 \pm 15.8$ yrs (ranging from 22 to $85 \mathrm{yrs}$ ), and duration of hemodialysis was $4.0 \pm 3.5 \mathrm{yr}$. Primary glomerulonephritis (GN) was the most prevalent kidney disease in the HD group: 207 (61.4\%) developed ESRD as a result of GN, $51(15.1 \%)$ as a result of hypertensive nephropathy (HN), $36(10.7 \%)$ as a result of diabetic nephropathy (DN), $16(4.7 \%)$ as a result of congenital or inherited causes, $6(1.8 \%)$ as a result of systemic lupus erythematosus (SLE) and $21(6.2 \%)$ as a result of other causes. Additionally, 267 (79.2\%) of the patients had anemia, and 250 (74.2\%) had hypertension. The 404 healthy individuals were age and sex matched, with a mean age of $53.1 \pm 16.2$ yrs, and 254 (62.9\%) were males.

3.1. Genotyping of BER Polymorphisms in HD Patients. The frequencies of the OGG1 c.977C > G, MTH1 c.247G > A, MUTYH c.972G > C, and AluYb8MUTYH genotypes associated with HD were shown in Table 2. The distribution in the healthy controls of these polymorphisms was consistent with Hardy-Weinberg equilibrium $(P>0.05$ for all).

Compared to healthy controls, the distribution of the genotypes in OGG1 c.977 C > G (namely, CC, CG, and GG) and the allele frequencies were not significantly different in the patients $\left(P>0.05, \chi^{2}\right.$ test $)$. For the c. $247 \mathrm{G}>\mathrm{A}$ in $M T H 1$, the frequency of heterozygous MTH1 c.247 G > A was only $6.2 \%$ and $7.7 \%$ in the patients and controls $(P=0.444)$, while the homozygote was not detected. Thus, the MTH1 polymorphism (c.247 G > A) was not included in further analysis.

Interestingly, both of the polymorphisms in the MUTYH gene showed an individual risk effect for ESRD (Table 2). For MUTYH c.972 G > C, the distribution of the three genotypes, namely CC, CG, and GG, and the allele frequencies were significantly different in HD patients $(P=0.046$ and 0.026$)$ compared with healthy controls. Furthermore, the frequency of the MUTYH c.972GG genotype was statistically higher in the HD cases $(40.9 \%)$ than in the controls $(32.2 \%)$, and the OR of GG adjusted by age and gender was 1.46 (95\% CI: 1.08-1.98; $P=0.013$ ). For AluYb8MUTYH, the distribution of the three genotypes and alleles in the HD patients was almost identical to that in the controls. Compared to the $A / A$ genotype, the $A l u Y b 8 M U T Y H$ insertion carriers $(A / P$ or $P / P)$ were significantly higher in HD patients, and the OR was 1.40 (95\% CI, 1.03-1.90; $P=0.034$ ).

Regarding the effect of the MUTYH c.972GG genotype and the AluYb8MUTYH P allele on ESRD, a combined risk analysis was performed and shown in Table 3. Individuals carrying the MUTYH c.972GG genotype might have a higher risk for ESRD, and the OR of MUTYH c.972GG adjusted by age and gender was 2.23 (95\% CI: $1.37-3.64 ; P=$ 0.001) among those with the OGG1 c.977GG genotype. Meanwhile, the presence of MUTYH c.972GG also added to the risk of $A l u Y b 8 M U T Y H A / P$ or $P / P$ genotypes for ESRD development (OR, 1.46; 95\% CI, 1.07-1.99; $P=0.017)$.

3.2. BER Polymorphisms in the Patients with Different Clinical Characteristics. The HD patients were stratified into six subgroups on the basis of the primary diagnoses (i.e., GN, HN, DN, congenital or inherited causes, SLE, or other causes). Similar to ESRD, the effects of BER polymorphisms on HD risk were confirmed in the 207 patients with primary diagnosis of glomerulonephritis when compared to the whole cohort (Table 4). The frequency of the MUTYH c.972 G > C GG genotype was significantly higher in cases than in controls, and the OR was 1.75 (95\% CI: 1.24-2.47; $P=$ 0.001). The frequency of the MUTYH AluYb8MUTYH A/P or $P / P$ genotype was significantly higher in cases than in controls, and the OR was 1.73 (95\% CI: 1.20-2.52; $P=$ $0.003)$.

In addition, the association of BER polymorphisms with risk of $\mathrm{HD}$ complication status was further analyzed (Table 4). Among 267 patients with anemia, the frequency of MUTYH c.972G > C GG was markedly higher in patients than controls (42.7\% versus $32.2 \%$; OR $(95 \% \mathrm{CI})=1.57$ (1.14-2.16); $P=0.006)$, whereas the AluYb8MUTYH insertion $(A / P$ or $P / P)$ significantly increased the risk for patients with anemia $(73.4 \%$ versus $62.6 \%$; OR $(95 \% \mathrm{CI})=1.78$ (1.22-2.60); $P=0.003)$. A similar relationship was detected among 250 patients with hypertension. The frequency of MUTYH c.972GG carriers was higher in cases than controls (41.2\% versus $32.2 \%$; OR $(95 \% \mathrm{CI})=1.48(1.07-2.05) ; P=$ $0.019)$. The frequency of $A l u Y b 8 M U T Y H$ insertion carriers $(A / P$ or $P / P)$ was higher in cases than controls $(70.4 \%$ versus 62.6\%; OR $(95 \% \mathrm{CI})=1.42(1.01-1.99) ; P=0.042)$.

3.3. Predictor Effect of BER Polymorphisms to 8-OHdG. The 8-OHdG levels in leukocyte DNA were evaluated in 116 HD patients divided into different subgroups according to the polymorphism genotypes and compared (Figure 2(a)). The genotypic frequencies of the three polymorphisms were 
TABLE 2: Genotypes of OGG1, MTH1, and MUTYH and the risk for HD.

\begin{tabular}{|c|c|c|c|c|}
\hline & Patients $(n=337)$ & Controls $(n=404)$ & $P$ value $^{\mathrm{a}}$ & OR $(95 \% \mathrm{CI})$ \\
\hline OGG1 c. $977 \mathrm{C}>\mathrm{G}$ & & & 0.394 & \\
\hline $\mathrm{CC}$ & $56(16.6 \%)$ & $77(19.1 \%)$ & & \\
\hline CG & $160(47.5 \%)$ & $200(49.5 \%)$ & & \\
\hline GG & $121(35.9 \%)$ & $127(31.4 \%)$ & 0.199 & $1.22(0.90-1.66)$ \\
\hline $\mathrm{CC}$ or $\mathrm{CG}^{\mathrm{b}}$ & $216(64.1 \%)$ & $277(68.6 \%)$ & & 1.00 \\
\hline C allele & 0.404 & 0.438 & & 1.00 \\
\hline G allele & 0.596 & 0.562 & 0.180 & $1.15(0.94-1.42)$ \\
\hline MTH1 c.247 G > A & & & 0.444 & \\
\hline GG & $316(93.8 \%)$ & $373(92.3 \%)$ & & 1.00 \\
\hline GA & $21(6.2 \%)$ & $31(7.7 \%)$ & & \\
\hline AA & 0 & 0 & & \\
\hline $\mathrm{GA}$ or $\mathrm{AA}^{\mathrm{b}}$ & $21(6.2 \%)$ & $31(7.7 \%)$ & 0.444 & $0.80(0.45-1.42)$ \\
\hline G allele & 0.969 & 0.962 & & 1.00 \\
\hline A allele & 0.031 & 0.038 & 0.453 & $0.81(0.46-1.42)$ \\
\hline MUTYH c. $972 \mathrm{G}>\mathrm{C}$ & & & 0.046 & \\
\hline $\mathrm{CC}$ & $44(13.1 \%)$ & $63(15.6 \%)$ & & \\
\hline CG & $155(46.0 \%)$ & $211(52.2 \%)$ & & \\
\hline GG & $138(40.9 \%)$ & $130(32.2 \%)$ & 0.013 & $1.46(1.08-1.98)$ \\
\hline $\mathrm{CC}$ or $\mathrm{CG}^{\mathrm{b}}$ & $199(59.1 \%)$ & $274(67.8 \%)$ & & 1.00 \\
\hline $\mathrm{C}$ allele & 0.361 & 0.417 & & 1.00 \\
\hline G allele & 0.639 & 0.583 & 0.026 & $1.27(1.03-1.57)$ \\
\hline AluYb8MUTYH & & & 0.099 & \\
\hline$A / A$ & $101(30.0 \%)$ & $151(37.4 \%)$ & & 1.00 \\
\hline$A / P$ & $164(48.7 \%)$ & $172(42.6 \%)$ & & \\
\hline$P / P$ & $72(21.3 \%)$ & $81(20.0 \%)$ & & \\
\hline$A / P$ or $P / P^{b}$ & $236(70.0 \%)$ & $253(62.6 \%)$ & 0.034 & $1.40(1.03-1.90)$ \\
\hline$A$ allele & 0.543 & 0.587 & & 1.00 \\
\hline$P$ allele & 0.457 & 0.413 & 0.092 & $1.19(0.97-1.47)$ \\
\hline
\end{tabular}

Note: CI: confidence interval; OR: odds ratio. ${ }^{a} P$ value for comparison using $\chi^{2}$ test to assess correlation between HD risk and predicted high-risk $O G G 1$, MTH1, and MUTYH genotypes and alleles; ${ }^{b}$ genotypes were combined properly to assess their association with HD and the genotype 1.00 as the reference category.

similar between the 116 patients tested for leukocyte DNA 8OHdG levels and all 337 patients investigated in the present study. In a parallel investigation of healthy controls in our laboratory, the HD patients exhibited increased 8-OHdG levels compared to the healthy individuals [12].

For the OGG1 c.977 C > G polymorphism, the genotypic frequencies (CC/CG/GG ratios of $15.5 \% / 43.1 \% / 41.4 \%$ ) for the 116 patients whose leukocyte DNA 8-OHdG levels had been analyzed did not vary significantly from the whole study population of 337 patients (16.6\%/47.5\%/35.9\%). The leukocyte 8-OHdG levels for patients carrying GG (26.7 \pm $\left.4.7 / 10^{6} \mathrm{dG}\right)$ or CG $\left(26.6 \pm 5.5 / 10^{6} \mathrm{dG}\right)$ were significantly higher than the patients carrying CC $\left(18.4 \pm 8.9 / 10^{6} \mathrm{dG}\right)$ $(P<0.001$ via ANOVA $)$. For the MUTYH c. $972 \mathrm{G}>\mathrm{C}$ polymorphism, the genotypic frequencies (CC/CG/GG ratios of $13.8 \% / 49.1 \% / 37.1 \%$ ) for the 116 patients did not vary significantly from the whole study population. The leukocyte 8-OHdG levels for patients carrying GG $\left(27.6 \pm 5.5 / 10^{6} \mathrm{dG}\right)$ or CG $\left(25.3 \pm 6.1 / 10^{6} \mathrm{dG}\right)$ were significantly higher than the patients carrying CC $\left(19.5 \pm 7.1 / 10^{6} \mathrm{dG}\right)(P<0.001$ via ANOVA). For the AluYb8MUTYH polymorphism, out of the 116 patients, 37, 52, and 27 showed the $A / A, A / P$ and $P / P$ genotypes, which did not differ from the whole population. The patients carrying $P / P\left(29.2 \pm 3.9 / 10^{6} \mathrm{dG}\right)$ or $A / P(25.4 \pm$ $5.6 / 10^{6} \mathrm{dG}$ ) had significantly higher 8 -OHdG levels than the patients carrying $A / A\left(22.6 \pm 7.9 / 10^{6} \mathrm{dG}\right)(P<0.001$ via ANOVA).

The combined impacts of these polymorphisms on 8OHdG levels were further investigated (Figures 2(b) and $2(\mathrm{c}))$. Based on the risk for HD, 43 patients carrying the MUTYH c.972GG genotype were analyzed; 6, 21, and 16 showed the CC, CG, and GG genotypes of OGG1 c.977C > G, respectively (Figure 2(b)). The OGG1 c.977C > G GG or CG genotypes significantly increased the 8-OHdG level when compared with patients with the OGG1 c.977C > G CC genotype among patients with the MUTYH c.972GG genotype $\left(29.2 \pm 3.2 / 10^{6} \mathrm{dG}, 28.1 \pm 4.8 / 10^{6} \mathrm{dG}\right.$ versus $21.8 \pm$ $9.0 / 10^{6} \mathrm{dG} ; P=0.01$ via ANOVA). This indicates that 


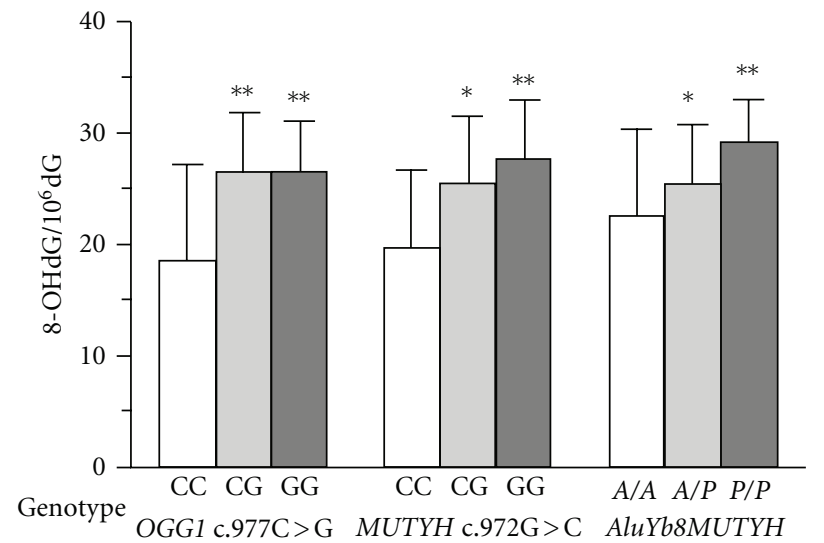

(a)

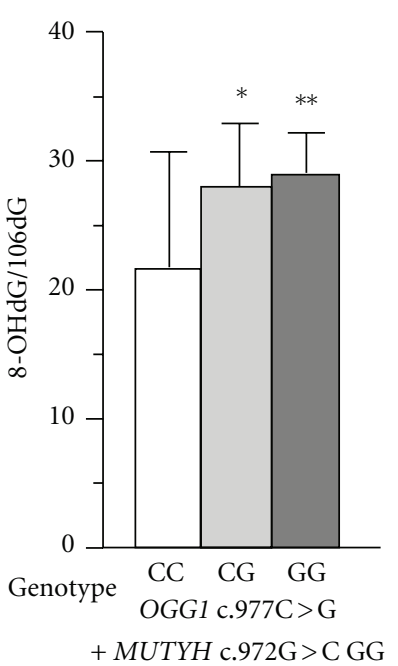

(b)

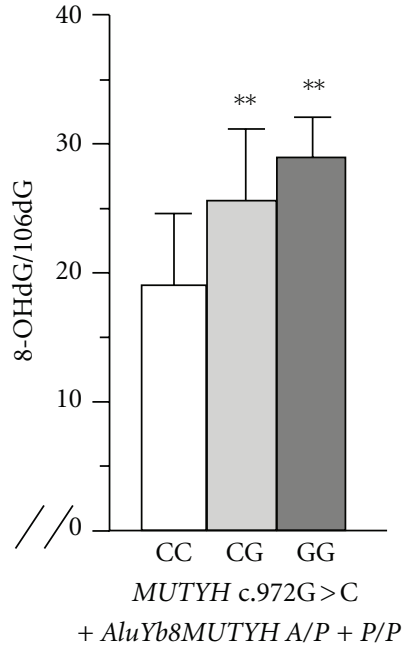

(c)

FIgUre 2: Individual (a) and combined [(b) accompanied with MUTYHc.972GG genotype; (c) accompanied with AluYb8MUTYH A/P or P/P genotype] analysis of the effect of BER polymorphism on mean levels of leukocyte DNA 8-OHdG in 116 HD patients. Every group includes three different bars stratified according to the polymorphism genotypes. Statistical significance was calculated using one-way ANOVA testing followed by post hoc analysis. ${ }^{*} P<0.05$ and ${ }^{* *} P<0.01$ versus subjects with the genotype in the blank bar.

TABLE 3: Combined analysis of genetic polymorphisms in OGG1 and MUTYH with HD risk.

\begin{tabular}{|c|c|c|c|c|}
\hline Genotypes & Patients $(n=337)$ & Controls $(n=404)$ & $P$ value ${ }^{\mathrm{c}}$ & OR $(95 \% \mathrm{CI})$ \\
\hline \multicolumn{5}{|c|}{ MUTYH c.972GG ${ }^{\mathrm{a}}$} \\
\hline \multicolumn{5}{|c|}{ OGG1 } \\
\hline c.977CC & $23(6.8 \%)$ & $27(6.7 \%)$ & 0.939 & $1.02(0.58-1.82)$ \\
\hline c.977CG & $67(19.9 \%)$ & $75(18.6 \%)$ & 0.650 & $1.09(0.75-1.57)$ \\
\hline c.977GG & $48(14.2 \%)$ & $28(6.9 \%)$ & 0.001 & $2.23(1.37-3.64)$ \\
\hline \multicolumn{5}{|c|}{ AluYb8MUTYH } \\
\hline$A / A$ & $18(5.3 \%)$ & $19(4.7 \%)$ & 0.691 & $1.14(0.59-2.22)$ \\
\hline$A / P$ & $58(17.2 \%)$ & $54(13.4 \%)$ & 0.146 & $1.35(0.90-2.02)$ \\
\hline$P / P$ & $62(18.4 \%)$ & $57(14.1 \%)$ & 0.113 & $1.37(0.93-2.03)$ \\
\hline \multicolumn{5}{|c|}{ AluYb8MUTYHA/P or $P / P^{b}$} \\
\hline \multicolumn{5}{|c|}{ OGG1 } \\
\hline c. $977 \mathrm{CC}$ & $38(11.3 \%)$ & $42(10.4 \%)$ & 0.701 & $1.10(0.69-1.74)$ \\
\hline c.977CG & $120(35.6 \%)$ & $136(33.7 \%)$ & 0.579 & $1.09(0.80-1.48)$ \\
\hline c.977GG & $78(23.1 \%)$ & $75(18.6 \%)$ & 0.125 & $1.32(0.93-1.89)$ \\
\hline \multicolumn{5}{|l|}{ MUTYH } \\
\hline c. $972 \mathrm{CC}$ & $11(3.3 \%)$ & $12(3.0 \%)$ & 0.818 & $1.10(0.48-2.53)$ \\
\hline c.972CG & $105(31.2 \%)$ & $130(32.2 \%)$ & 0.766 & $0.95(0.70-1.30)$ \\
\hline c.972GG & $120(35.6 \%)$ & $111(27.5 \%)$ & 0.017 & $1.46(1.07-1.99)$ \\
\hline
\end{tabular}

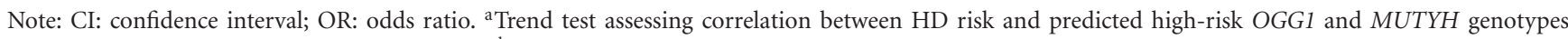
combined with the MUTYH c.972GG genotype. ${ }^{\mathrm{b}}$ Trend test assessing correlation between HD risk and predicted high-risk OGG1 and MUTYH genotypes combined with the AluYb8MUTYH A/P or $P / P$ genotype. c: $P$ value for comparison using $\chi^{2}$-test between patients and controls.

MUTYH and OGG1 may have synergistic roles in the prevention of DNA oxidative damage. Similarly, out of 79 patients carrying the $A l u Y b 8 M U T Y H$ insertion $(A / P$ or $P / P)$, 6, 41, and 32 showed the MUTYH c.972G > C CC, CG, and GG genotypes, respectively (Figure 2(c)). The 8-OHdG levels of individuals carrying the GG or CG genotypes were higher than in individuals carrying the CC genotype among patients with the AluYb8MUTYH insertion $\left(29.5 \pm 3.4 / 10^{6} \mathrm{dG}, 25.6 \pm\right.$ $5.3 / 10^{6} \mathrm{dG}$ versus $19.4 \pm 5.3 / 10^{6} \mathrm{dG} ; P<0.001$ via ANOVA).

\section{Discussion}

End-stage renal disease (ESRD) is a troublesome health problem worldwide, and the mortality rate for ESRD patients 


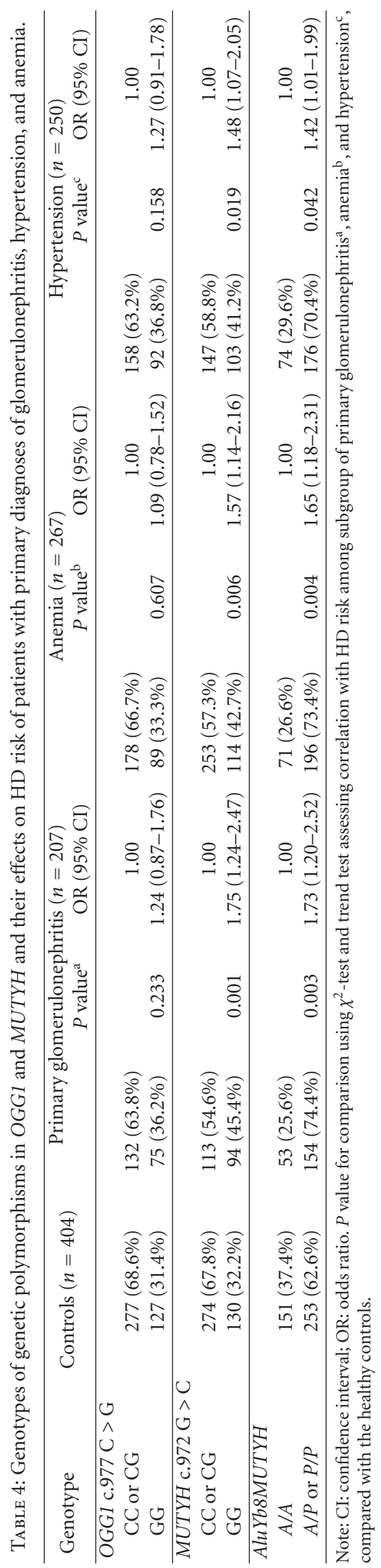


is 10 to 20 times higher than similarly aged individuals from the general population [22]. Maintenance hemodialysis (HD) is an efficient way to treat ESRD, and its use is increasing due to the epidemic of ESRD. New epidemiological studies show that China is also anticipating an increasing burden from ESRD and HD in the near future, although it used to be severely underestimated. It was reported that the number of patients with chronic kidney disease was 119.5 million in China [23], and the annual incidence of HD was estimated to be as high as 36.1 per million population (pmp) [24].

In this study, we investigated polymorphisms of base excision repair (BER) genes in a case-control Chinese population and demonstrated that individual and combined BER variations, mainly MUTYH polymorphisms, might increase the risk for ESRD. The underlying mechanical linkage might be an increase in 8-OHdG levels in leukocyte DNA, which was confirmed to be genetically determined. Oxidative DNA damage is unavoidable and is continuously generated by oxidative byproducts of normal cellular metabolism. The BER pathway is a critical process for genomic maintenance, as highlighted by the severe phenotypes seen in cells and animals deficient in BER function. MUTYH and OGG1 double knockout cells are more sensitive to oxidants, and the double knockout resulted in a reduction of $S$ phase and an increase in G2/M phase than wildtype cells, suggesting multiple roles of MUTYH and OGG1 in the maintenance of genome stability [25].

The effectiveness of DNA repair is subject to modulation by gene polymorphism. In the hemodialysis population, OGG1 c.977C > G, MUTYH c.972G > C, and AluYb8MUTYH showed significant effects on 8-OHdG levels in peripheral leukocytes, both individually and in combination. Our previous study illustrated that leukocyte 8OHdG levels are variable among the Chinese population, regardless of AluYb8MUTYH variations [12, 13]. In this study, we confirmed a relationship among ESRD patients. Patients carrying the OGG1 c.977 GG or CG genotypes had significantly higher 8-OHdG levels than those with the OGG1 c.977CC genotype. Similarly, Kohno et al. previously reported that 326Ser-containing (c.977CC) OGG1 has a seven-fold higher activity for repairing 8-oxoguanine than 326Cys-containing (c.977GG) OGG1 [26]. In a background of the MUTYH c.972GG genotype, OGG1 c.977C > G still showed a significant increase in 8 -OHdG levels in peripheral leukocytes. The same results were detected for the MUTYH c. $972 \mathrm{C}>\mathrm{G}$ combined with the AluYb8MUTYH $P$ allele. However, the patients carrying MUTYH c.972GG had significantly higher 8 -OHdG levels than the patients carrying MUTYH c.972CC. In contrast, Ali et al. reported that the glycosylase and DNA-binding activity was partially impaired in the MUTYH c.972CC genotype [27], whereas Shinmura and Yokota showed the same activity levels despite variation in the MUTYH c.972 G > C polymorphism [28]. Therefore, the genetic variations in the BER pathway may be enough to maintain 8-OHdG levels in nuclear DNA, although the underlying mechanisms are not extensively studied or understood.

Intriguingly, the genotype frequencies of $M U T Y H$ c.972GG or AluYb8MUTYH carriers $(A / P$ or $P / P)$ in the HD patients were markedly higher than those in the controls. The combined analysis showed that the risk of HD was further increased among the individuals carrying both the MUTYH c.972GG and OGG1 c.977GG genotypes as well as those with both the AluYb8MUTYH $(A / P$ or $P / P)$ and MUTYH c.972GG genotypes. The findings from Trabulus et al. [17] confirmed the association between DNA repair gene polymorphisms and ESRD development in Turkish population. And the combined effect of DNA repair variants added to such association, which was similarly illustrated in the present study.

It has been noted that the disease profile of ESRD is different in China from Western countries. Zuo and Wang showed that the glomerulonephritis remained the leading cause and accounted for nearly $50 \%$ of cases [24]. In this study, $61.4 \%$ of HD cases were the result of primary glomerulonephritis (GN). Additionally, the MUTYH GG genotype also significantly increased the risk for ESRD from GN $(\mathrm{OR}=1.75)$. This association remained in persons with the AluYb8MUTYH P allele, which increased the risk for ESRD from GN by a factor of 1.73. Thus, MUTYH c.972GG or AluYb8MUTYH could be the novel genetic risk factor for ESRD, and screening for these genetic variants or combined analysis may have predictive value in assessing potential risk in China.

Based on the correlation between BER polymorphisms and 8-OHdG levels, patients with different BER genetic polymorphisms were found to be at increased risk of cumulative oxidative DNA damage. Thus, we proposed that the relationship between genetic factors and ESRD and the effect of increased 8-OHdG levels underly this process. Increasing evidence has shown that the accumulation of 8OHdG in DNA could increase the risk of DNA mutations and cancer development $[29,30]$. We have also demonstrated that increased DNA oxidation might contribute to agerelated diseases [12]. 8-OHdG levels in leukocyte DNA of HD patients are significantly higher than healthy controls, which has been confirmed by other groups [31, 32]. However, to date, there has been no direct evidence demonstrating a cause-and-effect relationship between oxidative DNA damage and the development of GN and ESRD. Our study using genetic analysis supports such a relationship, but further studies are needed to elucidate the pathologic significance of oxidative DNA damage among people with respect to ESRD development.

Anemia and hypertension are the most frequent complications of ESRD and related to the increased mortality rates [33, 34]. Increased DNA damage is responsible for depressed production of erythropoietin (EPO), hypertension formation, and cardiovascular disease (CVD) [35, 36]. Observational studies have revealed a strong association between the severity of anemia and the risk of morbidity and mortality from cardiovascular disease and other causes in HD patients $[19,37]$; hypertension is also likely to be a major contributing factor to these events [38]. In this study, we illustrated that mutations in BER genes were tightly linked with the complications of anemia or hypertension among HD patients. As cardiovascular events are the primary cause of death in HD patients, these data suggest that oxidative 
DNA damage might be involved in the risk for complications and long-term outcomes.

However, not all relationships regarding BER polymorphisms in the present study can be explained thoroughly. It was demonstrated that the BER polymorphisms were related to the disability to repair oxidative DNA and then accumulation of the levels of 8-OHdG in leukocyte DNA. The high levels of 8-OHdG, therefore, contribute to the development of ESRD. The connection between genetic variations, oxidative DNA damage, and disease condition were not consistent. Take OGG1 c. $977 \mathrm{C}>\mathrm{G}$ for instance, the $G G$ and/or $C G$ variations significantly increased the 8OHdG levels, which predicts a high risk for oxidative DNA damage. But the OGG1 c.977C > G polymorphism did not appear to be related to ESRD among the investigated Chinese population. Tarng et al. also showed similar results among patients undergoing $\mathrm{HD}$, but did not provide a detailed interpretation [31].

In summary, our study showed that the polymorphisms in BER system, including MUTYH c.972GG and AluYb8MUTYH, increased the risk for ESRD development in China, especially their combined effect with $O G G 1$ c.977GG. It suggests that oxidative DNA damage might be one common risk factor for related renal diseases, and the genes in BER pathway may be involved in the progress of renal function deterioration and complications. Those homozygous or heterozygous for BER polymorphisms might be candidate genetic factors for ESRD development. Screening those polymorphisms would be helpful for preventing progression of chronic kidney disease and improving the patients' long-term outcomes of hemodialysis.

\section{Acknowledgments}

The authors are extremely grateful to Professor Daxi Ji and $\mathrm{Dr}$. Bin $\mathrm{Xu}$ for kind collaboration in recruitment of the investigated patients. This work was supported by the Jiangsu Science and Technology Foundation (Grant no. BK2009236), the National Natural Science Foundation of China (Grant nos. 81070579 and 81070273) and Open Foundation of State Key Laboratory of Pharmaceutical Biotechnology (Grant no. KF-GN-201201).

\section{References}

[1] S. Reuter, S. C. Gupta, M. M. Chaturvedi, and B. B. Aggarwal, "Oxidative stress, inflammation, and cancer: how are they linked?" Free Radical Biology and Medicine, vol. 49, no. 11, pp. 1603-1616, 2010.

[2] O. A. Sedelnikova, C. E. Redon, J. S. Dickey, A. J. Nakamura, A. G. Georgakilas, and W. M. Bonner, "Role of oxidatively induced DNA lesions in human pathogenesis," Mutation Research, vol. 704, no. 1-3, pp. 152-159, 2010.

[3] W. L. Neeley and J. M. Essigmann, "Mechanisms of formation, genotoxicity, and mutation of guanine oxidation products," Chemical Research in Toxicology, vol. 19, no. 4, pp. 491-505, 2006.

[4] C. J. Burrows and J. G. Muller, "Oxidative nucleobase modifications leading to strand scission," Chemical Reviews, vol. 98, no. 3, pp. 1109-1151, 1998.
[5] Y. Nakabeppu, "Regulation of intracellular localization of human MTH1, OGG1, and MYH proteins for repair of oxidative DNA damage," Progress in Nucleic Acid Research and Molecular Biology, vol. 68, pp. 75-94, 2001.

[6] R. Hakem, "DNA-damage repair; the good, the bad, and the ugly," EMBO Journal, vol. 27, no. 4, pp. 589-605, 2008.

[7] A. B. Robertson, A. Klungland, T. Rognes, and I. Leiros, "DNA repair in mammalian cells: base excision repair: the long and short of it," Cellular and Molecular Life Sciences, vol. 66, no. 6, pp. 981-993, 2009.

[8] S. Maynard, S. H. Schurman, C. Harboe, N. C. de Souza-Pinto, and V. A. Bohr, "Base excision repair of oxidative DNA damage and association with cancer and aging," Carcinogenesis, vol. 30, no. 1, pp. 2-10, 2009.

[9] R. J. Hung, J. Hall, P. Brennan, and P. Boffetta, "Genetic polymorphisms in the base excision repair pathway and cancer risk: a huge review," American Journal of Epidemiology, vol. 162, no. 10, pp. 925-942, 2005.

[10] M. Kasahara, K. Osawa, K. Yoshida et al., "Association of MUTYH Gln324His and APEX1 Asp148Glu with colorectal cancer and smoking in a Japanese population," Journal of Experimental and Clinical Cancer Research, vol. 27, no. 1, article 49, 2008.

[11] L. Le Marchand, T. Donlon, A. Lum-Jones, A. Seifried, and L. R. Wilkens, "Association of the hOGG1 Ser326Cys polymorphism with lung cancer risk," Cancer Epidemiology Biomarkers and Prevention, vol. 11, no. 4, pp. 409-412, 2002.

[12] C. Sun, H. Chen, W. Guo et al., "A common mutation of the $M Y H$ gene is associated with increased DNA oxidation and age-related diseases," Free Radical Biology and Medicine, vol. 48, no. 3, pp. 430-436, 2010.

[13] H. Chen, C. Sun, W. Guo et al., "AluYb8 insertion in the MUTYH gene is related to increased $8-\mathrm{OHdG}$ in genomic DNA and could be a risk factor for type 2 diabetes in a Chinese population," Molecular and Cellular Endocrinology, vol. 332, no. 1-2, pp. 301-305, 2011.

[14] J. Galle, "Oxidative stress in chronic renal failure," Nephrology Dialysis Transplantation, vol. 16, no. 11, pp. 2135-2137, 2001.

[15] A. B. Fogo and V. Kon, "The glomerulus-a view from the inside - the endothelial cell," International Journal of Biochemistry and Cell Biology, vol. 42, no. 9, pp. 1388-1397, 2010.

[16] T. Fukushima, T. Sasaki, and S. Arakawa, "hOGG1 polymorphism correlates with progression of IgA nephropathy (IgA GN)," Nephrology, vol. 6, supplement, pp. A10-A11, 2001.

[17] S. Trabulus, G. S. Guven, and M. R. Altiparmak et al., "DNA repair XRCC1 Arg399Gln polymorphism is associated with the risk of development of end-stage renal disease," Molecular Biology Reports, vol. 39, no. 6, pp. 6995-7001, 2012.

[18] P. Muntner, A. Anderson, J. Charleston et al., "Hypertension awareness, treatment, and control in adults with CKD: results from the chronic renal insufficiency cohort (CRIC) study," American Journal of Kidney Diseases, vol. 55, no. 3, pp. 441$451,2010$.

[19] F. Locatelli, R. L. Pisoni, C. Combe et al., "Anaemia in haemodialysis patients of five European countries: association with morbidity and mortality in the Dialysis Outcomes and Practice Patterns Study (DOPPS)," Nephrology Dialysis Transplantation, vol. 19, no. 1, pp. 121-132, 2004.

[20] S. A. Miller, D. D. Dykes, and H. F. Polesky, "A simple salting out procedure for extracting DNA from human nucleated cells," Nucleic Acids Research, vol. 16, no. 3, p. 1215, 1988.

[21] B. Halliwell, "Oxygen and nitrogen are pro-carcinogens. Damage to DNA by reactive oxygen, chlorine and nitrogen species: 
measurement, mechanism and the effects of nutrition," Mutation Research, vol. 443, no. 1-2, pp. 37-52, 1999.

[22] R. N. Foley, P. S. Parfrey, and M. J. Sarnak, "Clinical epidemiology of cardiovascular disease in chronic renal disease," American Journal of Kidney Diseases, vol. 32, no. 5, pp. S112-S119, 1998.

[23] L. Zhang, F. Wang, L. Wang et al., "Prevalence of chronic kidney disease in China: a cross-sectional survey," The Lancet, vol. 379, no. 9818, pp. 815-822, 2012.

[24] L. Zuo and M. Wang, "Current burden and probable increasing incidence of ESRD in China," Clinical Nephrology, vol. 74, pp. S20-S22, 2010.

[25] Y. Xie, H. Yang, J. H. Miller et al., "Cells deficient in oxidative DNA damage repair genes $M y h$ and $O g g 1$ are sensitive to oxidants with increased G2/M arrest and multinucleation," Carcinogenesis, vol. 29, no. 4, pp. 722-728, 2008.

[26] T. Kohno, K. Shinmura, M. Tosaka et al., "Genetic polymorphisms and alternative splicing of the hOGG1 gene, that is involved in the repair of 8-hydroxyguanine in damaged DNA," Oncogene, vol. 16, no. 25, pp. 3219-3225, 1998.

[27] M. Ali, H. Kim, S. Cleary, C. Cupples, S. Gallinger, and R. Bristow, "Characterization of mutant MUTYH proteins associated with familial colorectal cancer," Gastroenterology, vol. 135, no. 2, pp. 499-507, 2008.

[28] K. Shinmura and J. Yokota, "The OGG1 gene encodes a repair enzyme for oxidatively damaged DNA and is involved in human carcinogenesis," Antioxidants and Redox Signaling, vol. 3, no. 4, pp. 597-609, 2001.

[29] S. S. David, V. L. O'Shea, and S. Kundu, "Base-excision repair of oxidative DNA damage," Nature, vol. 447, no. 7147, pp. 941-950, 2007.

[30] B. van Loon, E. Markkanen, and U. Hübscher, "Oxygen as a friend and enemy: how to combat the mutational potential of 8-oxo-guanine," DNA Repair, vol. 9, no. 6, pp. 604-616, 2010.

[31] D. C. Tarng, T. J. Tsai, W. T. Chen, T. Y. Liu, and Y. H. Wei, "Effect of human OGG1 1245C $\rightarrow$ G gene polymorphism on 8-hydroxy-2'-deoxyguanosine levels of leukocyte DNA among patients undergoing chronic hemodialysis," Journal of the American Society of Nephrology, vol. 12, no. 11, pp. 2338-2347, 2001.

[32] Y. S. Lin, S. C. Hung, Y. H. Wei, and D. C. Tarng, "GST M1 polymorphism associates with DNA oxidative damage and mortality among hemodialysis patients," Journal of the American Society of Nephrology, vol. 20, no. 2, pp. 405-415, 2009.

[33] C. T. Jurkovitz, J. L. Abramson, L. V. Vaccarino, W. S. Weintraub, and W. M. McClellan, "Association of high serum creatinine and anemia increases the risk of coronary events: results from the prospective community-based atherosclerosis risk in communities (ARIC) study," Journal of the American Society of Nephrology, vol. 14, no. 11, pp. 2919-2925, 2003.

[34] S. Taddei, R. Nami, R. M. Bruno, I. Quatrini, and R. Nuti, "Hypertension, left ventricular hypertrophy and chronic kidney disease," Heart Failure Reviews, vol. 16, no. 6, pp. 615-620, 2011.

[35] N. R. Madamanchi, A. Vendrov, and M. S. Runge, "Oxidative stress and vascular disease," Arteriosclerosis, Thrombosis, and Vascular Biology, vol. 25, no. 1, pp. 29-38, 2005.

[36] E. L. Schiffrin, "Oxidative stress, nitric oxide synthase, and superoxide dismutase: a matter of imbalance underlies endothelial dysfunction in the human coronary circulation," $\mathrm{Hy}$ pertension, vol. 51, no. 1, pp. 31-32, 2008.

[37] B. M. Robinson, M. M. Joffe, J. S. Berns, R. L. Pisoni, F. K. Port, and H. I. Feldman, "Anemia and mortality in hemodialysis patients: accounting for morbidity and treatment variables updated over time," Kidney International, vol. 68, no. 5, pp. 2323-2330, 2005.

[38] M. V. Rocco, G. Yan, R. J. Heyka, R. Benz, and A. K. Cheung, "Risk factors for hypertension in chronic hemodialysis patients: baseline data from the HEMO study," American Journal of Nephrology, vol. 21, no. 4, pp. 280-288, 2001. 


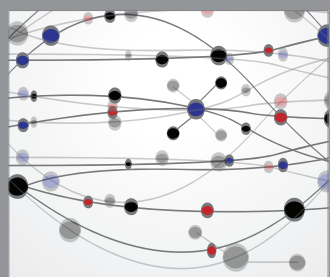

The Scientific World Journal
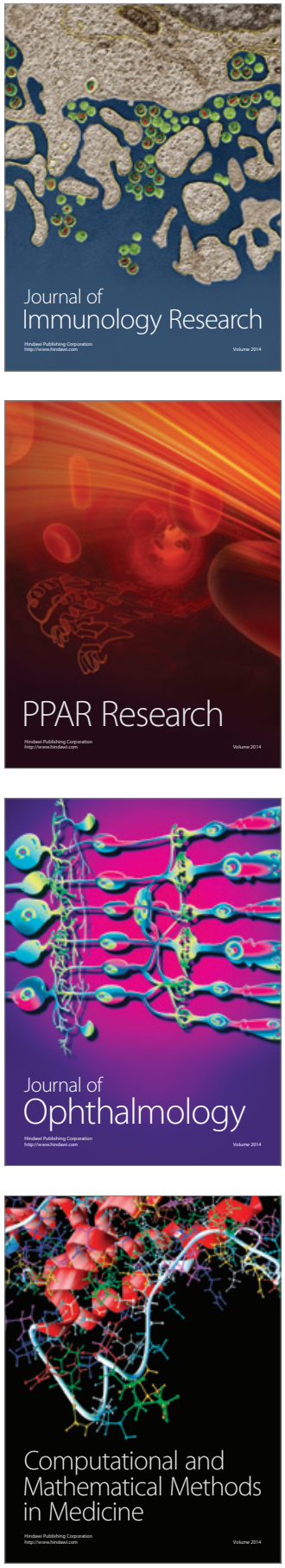

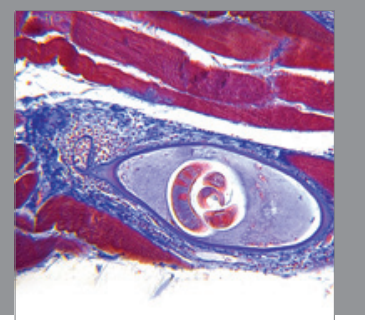

Gastroenterology

Research and Practice
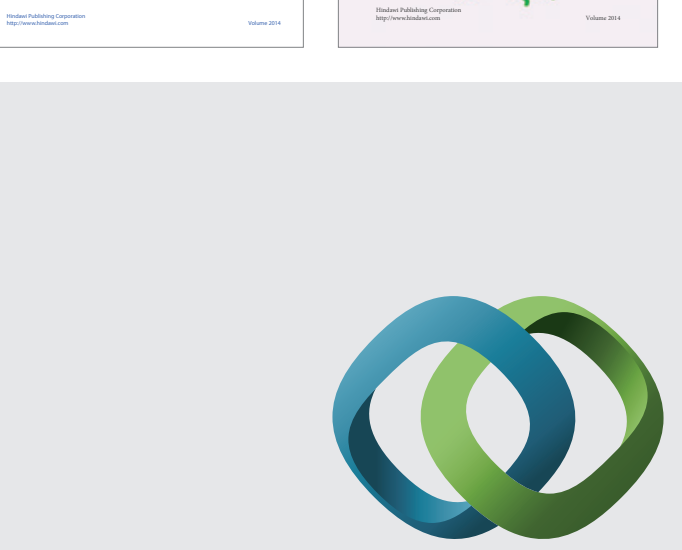

\section{Hindawi}

Submit your manuscripts at

http://www.hindawi.com
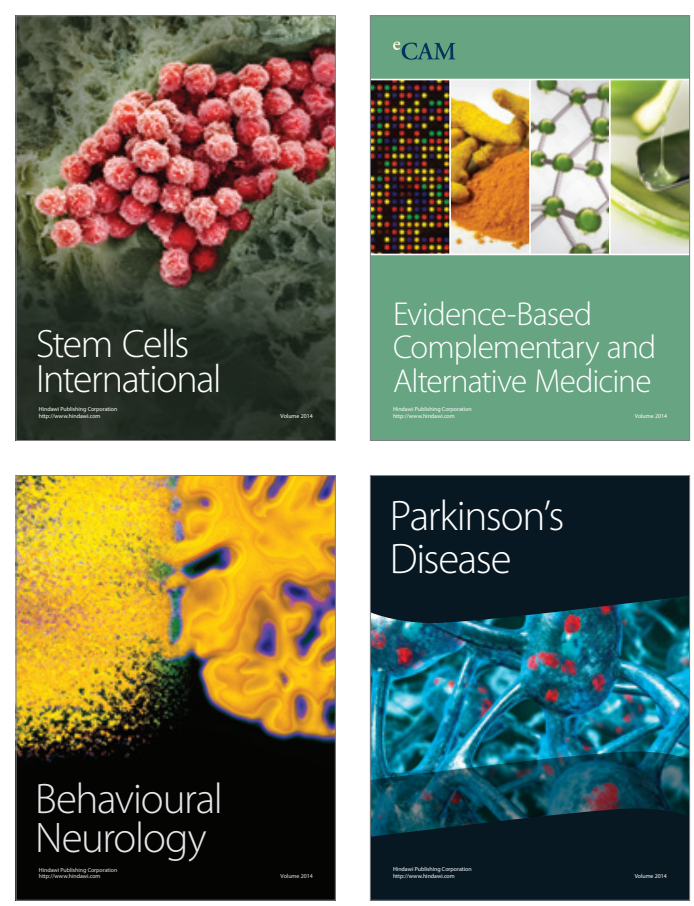

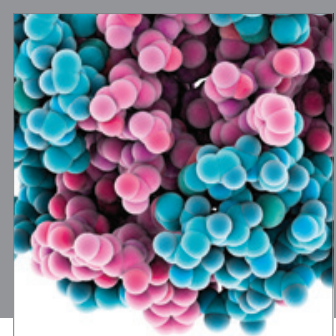

Journal of
Diabetes Research

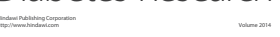

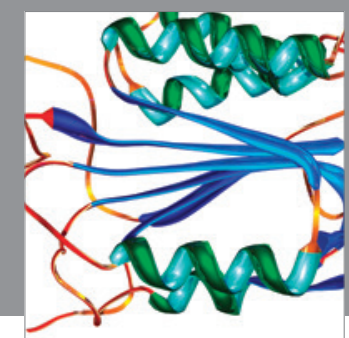

Disease Markers
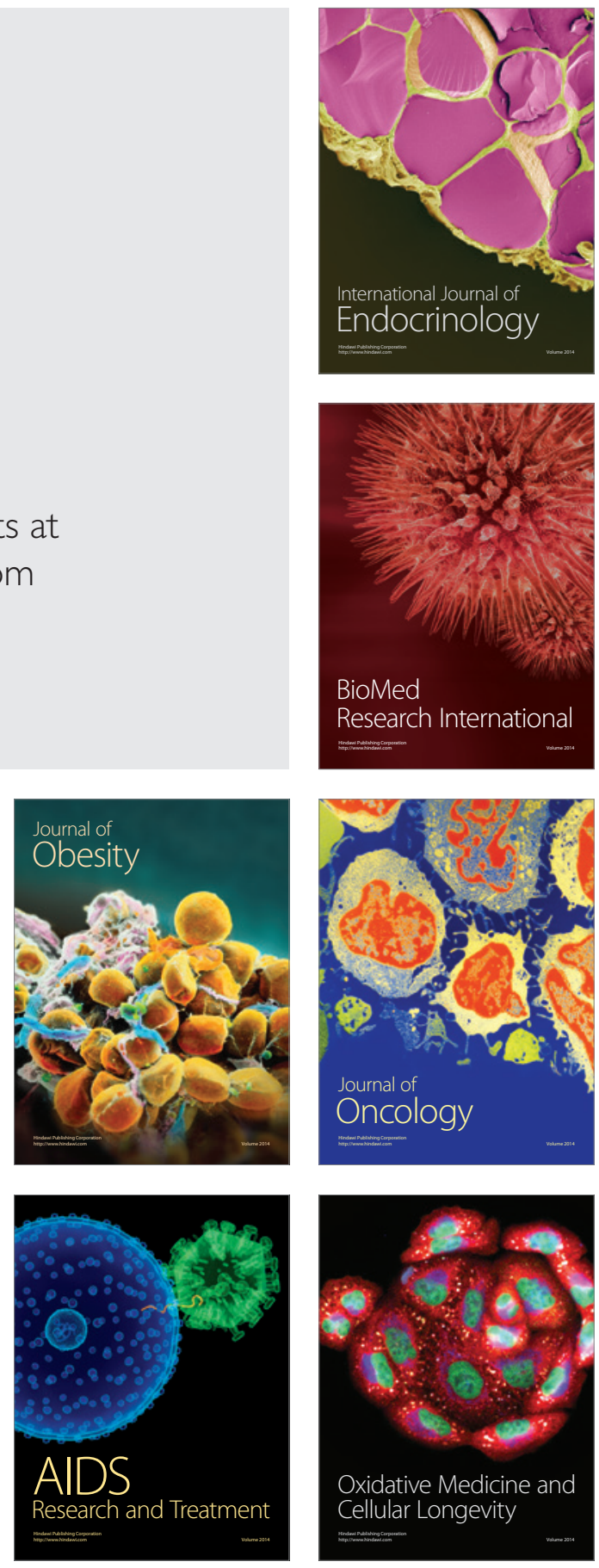